\section{Violência intrafamiliar: a experiência dos profissionais de saúde nas Unidades de Saúde da Família de São Joaquim do Monte, Pernambuco*}

\section{Intra-family violence: health workers' practice in São Joaquim do Monte, Pernambuco, Brazil}

\section{Marluce Tavares de Oliveira' \\ Isabella Samico"}

Ana Beatriz Matos Ishigami"'

Renata Maria Moura Nascimento"II

' Faculdade de Ciências Médicas da Faculdade de Ciências Médicas da Universidade de Pernambuco.

"Instituto de Medicina Integral Prof. Fernando Figueira.

"I' Residência de Saúde Coletiva da Faculdade de Ciências Médicas da Universidade de Pernambuco.

*Financiamento: CNPq: Edital 22/07 - Saúde da Mulher (Processo No. 551334/2007-0). FACEPE: Edital PPSUS-PE 2008 (Processo No. APQ-1373-4.00/08)

Correspondência: Marluce Tavares de Oliveira. Departamento de Medicina Social, Faculdade de Ciências Médicas, Universidade de Pernambuco. Rua Prof. Arnóbio Marques, 310, Santo Amaro, Recife, PE CEP 50.100-130. E-mail: tavaresm2002@yahoo.com.br

\section{Resumo}

O objetivo do estudo é verificar a associação entre atendimento, encaminhamento e notificação de violência intrafamiliar/ VIF contra adolescentes grávidas e características sociodemográficas de 84 profissionais vinculados ao Programa de Saúde da Família/PSF no agreste pernambucano. Trata-se de recorte de projeto multicêntrico, com desenho de corte transversal, realizado entre outubro de 2008 e abril de 2009. Foi aplicado um questionário estruturado referente às diversas manifestações da violência (psicológica, física ou sexual) e validado para o Brasil, ao qual foram acrescidas variáveis sociodemográficas e da experiência de atendimento de situações deVIF: formas identificadas, cuidados dispensados, encaminhamentos e notificação. A análise dos dados evidencia o grande número de profissionais que nunca identificaram (72\%) casos de violência. Entre os 23 que relataram atendimento, predominou a forma psicológica $(86,9 \%)$, seguida da física $(60,8 \%)$ e da sexual $(43,5 \%)$, sendo que apenas oito profissionais $(34,8 \%)$ encaminharam as adolescentes para Conselho Tutelar, Delegacia de Polícia e/ou Promotoria Pública. A análise da associação com atendimento e notificação relacionou aumento da idade $\left(\mathrm{p}=0,018 ; \mathrm{IC}_{95}\right.$ $1,31-17,1)$ e ter filhos ( $\mathrm{p}=0,039 ; \mathrm{IC}_{95} 0,04$ - 17,1) como sendo estatisticamente significante, enquanto trabalhar na área urbana e ter nível educacional superior de ocupação mostraram-se limítrofes. Sexo, situação marital e tempo na ocupação não mostraram associação. Conclui-se que, a despeito da “invisibilidade' da VIF, a análise das ações empreendidas no PSF representou uma aproximação deste fenômeno, complexo e multifacetado, ampliando as possibilidades de reflexão crítica sobre as diferentes formas de apreensão social do mesmo.

Palavras-chave: Violência intrafamiliar. Gestação. Adolescência. Profissionais de saúde. Fatores associados. 


\section{Abstract}

The objective of the study was to verify the association between assistance, referral and reporting of Intra-family Violence/ IFV against pregnant adolescents, and the socio-demographic characteristics of 84 professionals of the Family Health Program/ FHP in the agreste region of Pernambuco. The study is part of a multicenter, cross sectional research, carried out from October 2008 to April 2009. A structured questionnaire related to the various types of violence (psychological, physical or sexual) and validated for Brazil was used. Sociodemographic variables and professional experience in assisting cases of IFV were added to the instrument: identification of types, care, referral, and notification. The analysis of results showed a large number of professionals that had never identified a case of violence (72\%). Among the 23 who provided care to cases of violence, there was a majority of psychological (86.9\%), followed by physical (60.8\%), and sexual (43.5\%) cases. Only eight professionals (34.8\%) referred the adolescents to the Tutelary Council, Police Office and/or Public Prosecutor's Office. The analysis of association with care and reporting found older age $\left(\mathrm{p}=0.018 ; \mathrm{IC}_{95}\right.$ $1.31-17.1)$ and having children $(\mathrm{p}=0.039$; $\left.\mathrm{IC}_{95} 0.04-17.1\right)$ as statistically significant. On the other hand, a borderline association was found with work in an urban area and higher education. There was no association with sex, marriage status and years of work variables. The authors concluded that, despite the 'invisibleness' of IFV, the analysis of actions developed at the FHP represented an approximation to this complex and multifaceted phenomenon and the possibility of critical thinking on its different ways of social understanding.

Keywords: Intra-family violence. Pregnancy. Adolescence. Health workers. Risk factors.

\section{Introdução}

Os problemas vinculados à escalada da violência nas sociedades atuais vem despertando preocupação crescente em vários setores, constituindo-se, desde a década de 70, em relevante problema de saúde pública nas grandes cidades. Afeta principalmente a população de adolescentes e adultos jovens, tornando-se uma das principais causas de morbimortalidade ${ }^{1-3}$. Embora não sendo um problema exclusivo da saúde, a violência traz influências marcantes e diretas sobre ela, provocando desde pequenas lesões até mortes. Insere-se nas relações dominantes ao se referir à hierarquia de poder estatal $\mathrm{e}$ dos conflitos de autoridade, disciplinando pessoas, famílias, comunidades e instituições, onde o desejo de domínio e aniquilamento do outro inviabiliza o estabelecimento de pactos de cidadania. Caracteriza-se, portanto, como um fenômeno de múltiplas causalidades ${ }^{4}$.

Descrita como "toda ação ou omissão que prejudique o bem-estar, a integridade física, psicológica ou a liberdade e o direito ao pleno desenvolvimento de um membro da família" ${ }^{5}$, a violência intrafamiliar é considerada mesmo quando praticada fora do domicílio da vítima, quer tenha sido ocasionada pelos pais biológicos ou por pessoas que exerçam a função parental $^{6}$. Conforme encontrado na literatura, a maioria dos casos de violência acontece no próprio domicílio, afetando principalmente crianças, mulheres e idosos, podendo gerar não só na vítima, mas em toda a sua família, traumas marcantes durante toda vida ${ }^{7}$. A violência contra crianças e adolescentes tem sido classificada como "todo ato ou omissão cometido por pais, parentes, outras pessoase instituições capazes de causar danos físico, sexual elou psicológico à vítima"'.

Enfocando especificamente a questão da violência doméstica durante a gravidez, agências públicas americanas (American Medical Association, Control Disease Center) passaram a enfatizar, na década de 90 , a atenção para abuso físico ou verbal das mulheres como problema de saúde públi- 
$\mathrm{ca}^{9}$, enquanto estudos realizados em países em desenvolvimento revelam Coeficientes de Incidência de violência na gestação que variam entre 4 a $29 \%{ }^{10}$.

No Brasil, estudo de base populacional empreendido com mulheres de 15 a 49 anos, residentes em S. Paulo e na zona da mata pernambucana ${ }^{11}$, encontrou prevalência de violência por parceiro íntimo de $28,9 \%$ e $36,9 \%$, respectivamente. Resultados preliminares* de pesquisa conduzida por Barreto, Ludermir e Valongueiro, envolvendo grávidas cadastradas no programa de Saúde da Família (PSF) de um distrito sanitário da cidade do Recife, mostram que em torno de $29 \%$ dessas mulheres sofreram algum tipo de agressão antes da gravidez. Ainda que a proporção de mulheres em situação de violência não tenha se alterado com a gravidez, houve uma inversão com relação ao tipo de agressão sofrida: a agressão física exclusiva ou associada a outro tipo de violência sofreu redução de $20 \%$ para $10 \%$ das mulheres, enquanto a frequência de violência psicológica elevou-se de $10 \%$ para $16 \%$ entre as entrevistadas.

No âmbito dos serviços de saúde, os reflexos da violência são nitidamente percebidos: seja pelos custos econômicos com assistência médica, tratamento e reabilitação das vítimas, custos com o sistema judiciário e penal, custos sociais decorrentes da queda de produtividade, além das lesões e traumas direta e indiretamente decorrentes do ato violento, que exercem um grande impacto em longo prazo ${ }^{7,12-14}$.

Dado o impacto da violência na trajetória de vida de crianças e adolescentes, o Estatuto da Criança e do Adolescente - ECA ${ }^{15}$, Lei 8.069/90, tornou obrigatória a notificação de casos suspeitos ou confirmados de maus-tratos contra estes grupos populacionais (Art.13), especificando penas aplicáveis ao médico, ao professor ou ao responsável por estabelecimento de atenção à saúde e de ensino fundamental, pré-escola ou creche que deixarem de comunicar à autoridade os casos de que tenham conhecimento (Art. 245).

Concorrem para os baixos índices de notificação em nosso país a falta de conscientização social dos profissionais, o desconhecimento das atitudes a serem tomadas diante dos casos, o medo do revanchismo por parte da família, o temor de transtornos legais ou de acusações de falsa denúncia, a precariedade e/ou ineficácia dos suportes sociais existentes, que colaboram para a descrença dos profissionais quanto ao desdobramento da notificação ${ }^{16}$.

Apesar de considerar o esforço do setor saúde em recomendar as ações e práticas de diagnóstico, tratamento e prevenção de violência doméstica e a fixação de diretrizes referentes à capacitação de recursos humanos em relação à implementação da política específica, as modificações curriculares nesta direção ainda são lentas, pontuais e pouco conhecidas ${ }^{17}$.

Os casos notificados apresentam grande importância, pois é por meio deles que a violência ganha visibilidade, permitindo o dimensionamento epidemiológico do problema e a criação de políticas públicas voltadas à sua prevenção. Conhecer serviços de atendimento psicossocial da rede de atendimento e fazer os necessários encaminhamentos é um passo importante para identificação do problema e de seu enfrentamento ${ }^{18}$.

Dessa maneira, o setor saúde e, em especial, as equipes de Saúde da Família/PSF, têm importante papel no enfrentamento da violência intrafamiliar, não subestimando a importância do fenômeno, habitualmente voltado apenas para as lesões físicas ${ }^{2,19}$. Esse fato pode estar relacionado à falta de preparo profissional ${ }^{14}$, ao desconhecimento da legislação de proteção, como o Estatuto da Criança e Adolescente ${ }^{15} \mathrm{e}$ a lei Maria da Penha ${ }^{20}$, ou simplesmente à decisão de não se envolver com os casos, sendo denominado um fenômeno invisível dentro dos serviços de saúde ${ }^{21}$.

*Lima H. Notícias. Ascom/UFPE, 2006. Disponível na internet em http://www.ufpe.br/new/visualizar.php?id=4611. [Acessado em janeiro de 2008] 
A educação permanente mantém estreita relação entre o processo formativo $\mathrm{e}$ o processo de trabalho em saúde ${ }^{22}$, porém observa-se hoje que a maioria das disciplinas da saúde não contempla em seus currículos e programas de educação continuada a formação e o treinamento dos aspectos relacionados com a violência. Por isso, os profissionais de saúde não se encontram preparados para oferecer uma atenção que tenha impacto efetivo à saúde das vítimas ${ }^{23}$. A partir da educação continuada, da ampliação de redes de suporte para profissionais e da melhoria de entes protetores à criança e ao adolescente, pode-se diminuir o grau de insegurança dos profissionais e, assim, aumentar o número de notificações de maus tratos.

O presente estudo teve por objetivo verificar a associação do atendimento, encaminhamento e notificação de violência intrafamiliar contra adolescentes grávidas realizados no PSF do município de São Joaquim do Monte, no agreste pernambucano, segundo características sociodemográficas dos profissionais de saúde.

\section{Metodologia}

Os dados utilizados fazem parte de um projeto multicêntrico mais amplo, envolvendo a avaliação da implantação de programa educativo-vivencial voltado para capacitação de profissionais da atenção básica, com vistas à identificação e ao acolhimento de gestantes adolescentes em situação de violência doméstica e familiar. O desenho adotado neste recorte foi de um estudo de corte transversal ${ }^{24}$, envolvendo o universo de profissionais vinculados ao Programa de Saúde da Família - PSF, do município de São Joaquim do Monte, no período de outubro de 2008 a abril de 2009. O município, localizado no agreste pernambucano, a $137 \mathrm{~km}$ da capital, com população de 21.442 habitantes (23\% de adolescentes) em uma área de $278 \mathrm{Km}^{2}$, de acordo com dados do IBGE para 2005; representa importante pólo agropecuário e religioso do Estado, com grandes desigualdades sociais ${ }^{25}$. Do ponto de vista da assistência à saúde, a totalidade da população está coberta pelo PSF (95\%) e pelo Programa de Agentes Comunitários de Saúde - PACS (5\%), com quatro equipes na área urbana e quatro na área rural, sendo ínfima a parcela com plano privado de saúde $(0,7 \%)$.

$\mathrm{O}$ instrumento de coleta de dados utilizado foi um questionário estruturado. Para identificação de casos de violência atendidos, foi utilizada a resposta positiva a pelo menos um dos itens dos blocos de questões do instrumento VPI - Violência por parceiro Íntimo - referente às diversas manifestações da violência (psicológica, física ou sexual). Ao instrumento em questão, validado para o Brasil $^{26}$, foram acrescidas variáveis sociodemográficas e da experiência de atendimento de situações de violência intrafamiliar (VIF): formas identificadas, cuidados dispensados, encaminhamentos e notificação.

Quanto ao agressor, utilizou-se a relação de parentesco ou afinidade com a adolescente (pai, mãe, avós, tios, primos, parceiro íntimo e outros). Entendeu-se parceiro íntimo, como o companheiro, ex-companheiro, independente da existência de união formal, ou namorado atual com quem a adolescente mantenha relações sexuais $^{26}$

Previamente à entrada em campo foi realizada etapa de treinamento da equipe para padronização de procedimentos e instrumentos, por meio de reuniões do grupo operativo com uma das coordenadoras do projeto, e teste piloto do instrumento em pesquisa prévia, envolvendo profissionais do PSF de outro município. Embora mantida a leitura de acordo com a redação original das questões doVPI, em algumas perguntas as entrevistadoras, todas mulheres, foram orientadas a incluir sinônimos ou expressões regionalmente utilizadas, no sentido da melhora da compreensão dos enunciados. Desta forma, no bloco referente à violência psicológica; à expressão "insultou" se acresceu "xingou", enquanto ao termo "depreciou ou humilhou" acrescentou-se "fez pouco". No bloco da violência física, "surrou" foi complementado por "lhe deu 
uma pisa" e "estrangulou" foi adicionado de "apertou seu pescoço". Para a violência sexual, prática "degradante ou humilhante" foi explicada como "realizar ou praticar ato ou 'brincadeira' sexual da qual tivesse vergonha". A abordagem dos profissionais se deu no ambiente de trabalho, em momento e local considerado adequados pela dupla entrevistado-entrevistador, não havendo recusas. $\mathrm{O}$ controle de qualidade das entrevistas ficou sob a responsabilidade da supervisora de campo e incluiu a reaplicação do questionário a $20 \%$ da população participante do piloto.

A realização de reuniões de sensibilização para apresentação dos objetivos e etapas da pesquisa, convocada pela Coordenação da Atenção Básica com o aval da Secretaria Municipal de Saúde, reiterou o apoio do secretário de saúde ao projeto de pesquisa e favoreceu a adesão do universo de profissionais da rede local do PSF.

Os dados coletados foram processados no módulo "Enter" do programa Epi-info, 2000, versão para Windows, utilizando-se um questionário eletrônico semelhante ao instrumento de coleta, construído no "Eped". Todos os dados coletados foram processados por dois digitadores independentes e os bancos resultantes confrontados por meio do módulo "Validate".

No que se refere aos aspectos analíti$\cos ^{27}$, a principal medida de associação foi a Razão de Prevalência - RP, que mede a associação entre as prováveis situações de risco/exposição e o evento investigado-VIF, com seus respectivos intervalos de confiança - IC ao nível de 95\% (valor de $\mathrm{p} \leq 0,05$ ). Foi realizada a distribuição de frequência das variáveis estudadas. A seguir, as variáveis independentes foram descritas para os grupos afetados e não afetados pela VIF, analisando-se a diferença de proporções por meio do teste do qui-quadrado $\left(\chi^{2}\right)$, com correção de Yates, quando indicado, considerando o valor de $p \leq 0,05$. Utilizou-se, para o cálculo estatístico, o programa Epi-Info 2000 for windows, versão 3.4.

O estudo foi aprovado pelo Comitê de Ética em Pesquisa em Seres Humanos do
Instituto de Medicina Integral Prof. Fernando Figueira - IMIP (Processo no ${ }^{\circ}$ 1224/08), e os participantes assinaram termo de consentimento livre e esclarecido. No sentido de buscar suporte para emoções emergentes a partir dos relatos de violência vivenciadas, foram realizados contatos com profissionais do PSF e profissional de Psicologia que atende à demanda de saúde mental do município, além da disponibilização de espaços de acolhimento e escuta qualificada com utilização da Terapia Comunitária ${ }^{28}$ para profissionais e pesquisadoras, por parte da coordenação da equipe de campo, com formação em Terapia Familiar e Comunitária.

\section{Resultados}

Foram entrevistados 84 profissionais vinculados ao Programa de Saúde da Família do município de São Joaquim do Monte, no agreste pernambucano. Entretanto, dois questionários foram extraviados, sendo por isso os resultados referentes a 82 entrevistas $(97,6 \%)$. A população de estudo foi majoritariamente constituída por mulheres $(79,3$ $\%)$ até 40 anos (75\%), com companheiro/a $(58,5 \%)$ e filhos $(63,4 \%)$.

Segundo a unidade de saúde (USF) de alocação, 52,4\% são de área urbana e 47,6\% de área rural. Quanto à ocupação, 30,4\% dos profissionais exercem atividades de nível superior (médico, enfermeiro e odontólogo) e $56,1 \%$ são de nível médio (agentes comunitários - ACS e de consultório dental - ACD). A média do tempo na ocupação foi de 9,5 \pm 8,2 anos, com tempo mínimo de 6 meses e máximo de 40 anos (Tabela 1).

Dentre os 82 profissionais entrevistados, 23 referiram ter atendido algum caso de violência contra gestantes adolescentes (28\%) no tempo de trabalho na atividade. A forma psicológica se apresentou como a referência mais lembrada ( $87 \%$ dos relatos), embora os demais tipos detenham frequências expressivas. Foi comum referência a acometimento simultâneo por mais de um tipo de violência (Tabela 2).

Dos profissionais que atenderam violência, oito $(34,8 \%)$ encaminharam casos, com 
Tabela 1 - Caracterização sociodemográfica dos profissionais de saúde do Programa de Saúde da Família - PSF, São Joaquim do Monte - Pernambuco, 2009.

Table 1 - Socio-demographic data of professionals of the Family Health Program - FHP, São Joaquim do Monte - Pernambuco, 2009.

\begin{tabular}{|c|c|c|}
\hline Variáveis sociodemográficas & $\mathrm{n}$ & $\%$ \\
\hline \multicolumn{3}{|l|}{ Sexo } \\
\hline Masculino & 17 & 20,7 \\
\hline Feminino & 65 & 79,3 \\
\hline \multicolumn{3}{|l|}{ Faixa etária } \\
\hline 17 a 30 & 31 & 37,8 \\
\hline 31 a 40 & 30 & 36,6 \\
\hline 41 e mais & 21 & 25,6 \\
\hline \multicolumn{3}{|l|}{ Área da USF } \\
\hline Rural & 39 & 47,6 \\
\hline Urbana & 43 & 52,4 \\
\hline \multicolumn{3}{|l|}{ Profissão } \\
\hline Médico & 6 & 7,3 \\
\hline Enfermeiro & 12 & 14,6 \\
\hline Odontólogo & 7 & 8,5 \\
\hline ACS & 38 & 46,3 \\
\hline$A C D$ & 8 & 9,8 \\
\hline Auxiliar ou técnico de enfermagem & 11 & 13,4 \\
\hline \multicolumn{3}{|l|}{ Situação marital } \\
\hline União estável & 48 & 58,5 \\
\hline Sem companheiro & 34 & 41,5 \\
\hline \multicolumn{3}{|l|}{ Filhos } \\
\hline Sim & 52 & 63,4 \\
\hline Não & 30 & 36,6 \\
\hline Total & 82 & 100,0 \\
\hline
\end{tabular}

utilização predominante de instrumento escrito, seguido da comunicação telefônica ou verbal $(43,7 \%$ e $37,5 \%$, respectivamente). O Conselho Tutelar (06 citações), a Delegacia de Polícia (04 referências) e a Promotoria Pública (02 relatos) representaram as instâncias referidas, sinalizando para a utilização de mais de um locus de encaminhamento (Tabela 2).

Os motivos alegados para o não encaminhamento pelos outros 15 profissionais que informaram atendimento de gestantes adolescentes em situações de violência, foram: falta de instrumento (4/15); medo de retaliação por parte do agressor e falta de provas (três referências, cada) e desconhecimento da obrigatoriedade de notificar (2/15).

Em relação aos agressores (dados não apresentados em tabela), os profissionais de saúde relataram que em $52,2 \%$ dos casos o responsável foi o parceiro, seguido pelo pai e mãe da adolescente $(47,8 \%$ e $39,1 \%$, respectivamente).

Quando questionados acerca da experiência profissional, com ampliação da população usuária para crianças e adolescentes (dados não apresentados em tabela), independentemente de se constatar ou não gravidez, estes profissionais referiram um 
Tabela 2 - Frequência de atendimento, tipos de violência e conduta frente a casos de violência contra gestantes adolescentes pelos profissionais do Programa Saúde da Família - PSF, São Joaquim do Monte - Pernambuco, 2009.

Table 2 - Frequency distribution of identification of violence, type of violence and health care strategies for cases of violence against pregnant adolescents, by professionals of the Family Health Program - FHP, São Joaquim do Monte - Pernambuco, 2009.

\begin{tabular}{|c|c|c|}
\hline Variáveis & $\mathrm{n}$ & $\%$ \\
\hline \multicolumn{3}{|l|}{ Atendeu casos de violência } \\
\hline Sim & 23 & 28,0 \\
\hline Não & 59 & 72,0 \\
\hline \multicolumn{3}{|l|}{ Tipos de violência atendida' } \\
\hline Psicológica & 20 & 87,0 \\
\hline Física & 14 & 60,9 \\
\hline Sexual & 10 & 43,5 \\
\hline \multicolumn{3}{|l|}{ Encaminhou algum caso de violência } \\
\hline Sim & 08 & 34,8 \\
\hline Não & 15 & 65,2 \\
\hline \multicolumn{3}{|l|}{ Notificação de violência } \\
\hline Sim & 16 & 19,5 \\
\hline Não & 66 & 80,5 \\
\hline \multicolumn{3}{|l|}{ Instrumento para a notificação ${ }^{2}$} \\
\hline Comunicação verbal/telefônica & 06 & 37,5 \\
\hline Boletim de ocorrência & 01 & 6,3 \\
\hline Encaminhamento escrito & 07 & 43,7 \\
\hline Outros & 02 & 12,5 \\
\hline Total & 82 & 100,0 \\
\hline
\end{tabular}

'Os casos de violência não são mutuamente excludentes, sendo os percentuais calculados levando em conta o $n^{\circ}$. de profissionais que atendeu VIF ( $n=23) /$ ' Cases of violence are not mutually excluding, and the percentages calculated took into account the number of professionals who assisted intra-familiar violence (IFV) cases ( $n=23$ ).

${ }^{2}$ Percentuais calculados levando em conta os 16 profissionais que informaram notificação / 2 Percentages calculated taking into account 16 professionals that informed reporting.

total de 31 casos atendidos no último ano, sendo que a notificação foi referida por apenas um de cada cinco respondentes (19,5\%). Os motivos para a não notificação seguiram o mesmo perfil de distribuição dos casos envolvendo gestantes adolescentes.

$\mathrm{Na}$ análise da associação das variáveis de interesse do estudo (Tabela 3), verificou-se que o aumento da idade (profissionais $>40$ anos) está relacionado a uma maior frequência de referência a atendimento $\mathrm{e}$ notificação de casos de violência, sendo a associação estatisticamente significante $(\mathrm{p}=0,018$ e 0,011 ; respectivamente). Verificou-se que os profissionais de área urbana referem maior frequência de aten- dimento e notificação quando comparados aos profissionais que exercem atividades na área rural $(\mathrm{p}=0,057 \mathrm{e} 0,052$; respectivamente) e, quando avaliado o nível educacional da ocupação, os profissionais de nível superior demonstraram encaminhar e notificar casos de violência com maior frequência, quando comparados a profissionais de nível médio. Embora esses resultados sugiram uma associação, esta não foi estatisticamente significante $(\mathrm{p}=$ $0,104)$. A condição de ter filhos mostrou uma associação significativa com a notificação ( $p=0,039$ ), onde profissionais com filhos têm maior probabilidade de notificar um caso de violência. 


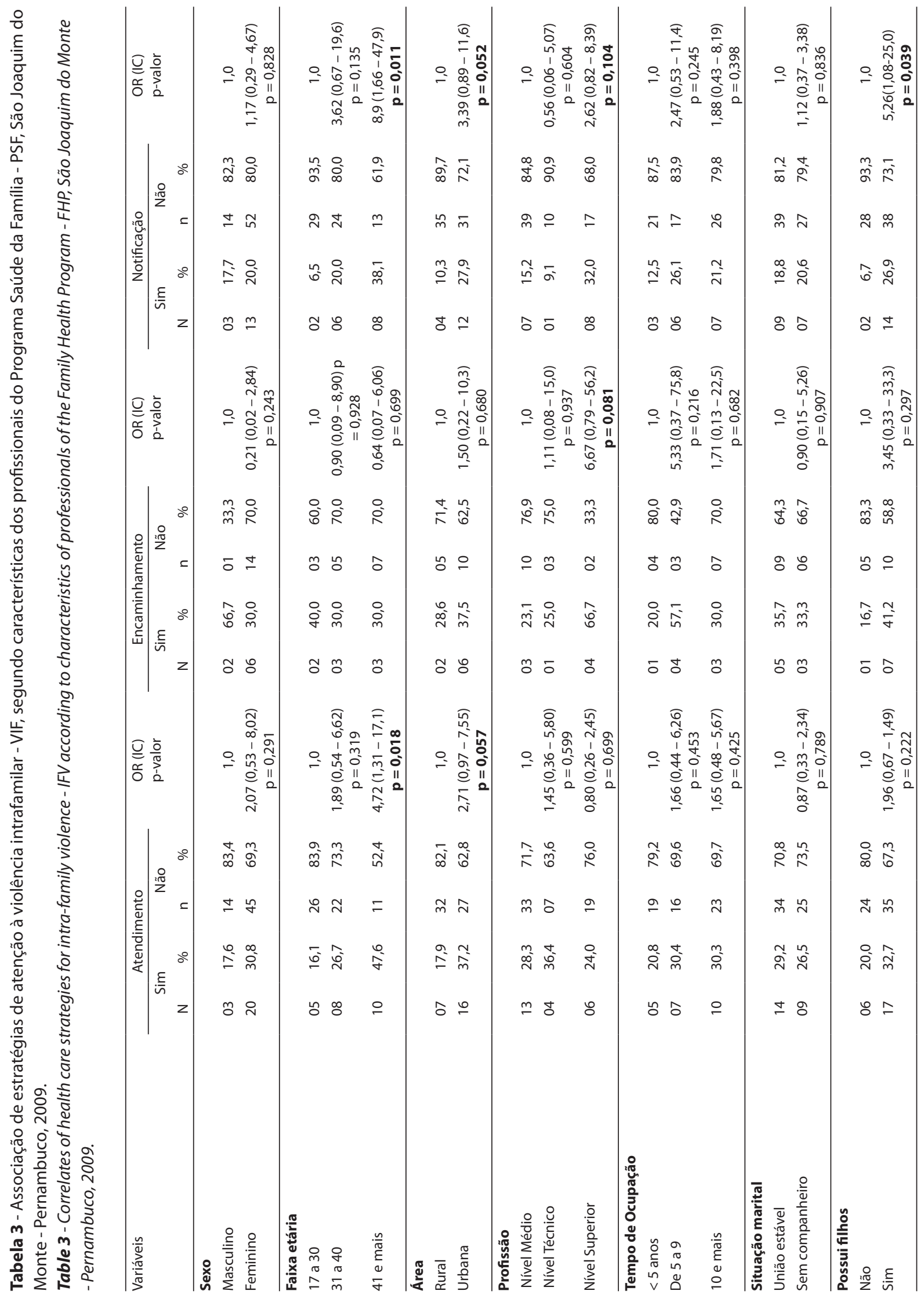




\section{Discussão}

A constituição da população de estudo majoritariamente formada por profissionais de nível médio e auxiliar, do sexo feminino até 40 anos, com companheiro/a e filhos, corresponde à distribuição habitual das equipes de saúde, entre elas o PSF, com marcante "feminilização" do setor ${ }^{29,30}$, o que pode produzir profundas alterações nas relações interpessoais e na vivência pessoal de situações de violência que impactam na identificação e cuidados dispensados, colocando em evidência o grande número de profissionais que nunca identificaram ( $72 \%$ da população investigada) e corroborando estudos empreendidos por vários autores ${ }^{2,31,32}$.

A predominância da forma psicológica $(86,9 \%)$, seguida da física $(60,8 \%)$ e da sexual (43,5\%), encontra correspondência com estudos realizados envolvendo mulheres de 15 a 49 anos $^{33}$, onde a violência psicológica por parceiro íntimo foi descrita como a forma de violência familiar mais frequente na população estudada (52\%), seguida pela física $(40,5 \%)$ e a sexual $(20,4 \%)$. Dados de estudo multicêntrico coordenado pela Organização Mundial da Saúde demonstraram que 15\% a $71 \%$ das mulheres reportaram terem sofrido atos de abuso emocional ${ }^{9}$.

Os patamares mais elevados encontrados em nossa pesquisa, em relação ao estudo já feito anteriormente ${ }^{33}$, podem estar relacionados tanto à diferença na população de estudo, representada por profissionais do PSF; à referência a acometimento simultâneo por mais de um tipo de violência; à maior participação do parceiro íntimo como agressor (52,2\%), como à possibilidade de ocorrência de viés de memória seletiva dos entrevistados pela maior permanência do impacto provocado por situações que envolvem agressões físicas, especialmente o abuso sexual contra adolescentes. A frequência de referência à violência e suas diferentes tipologias passa pela percepção pessoal e social do que é um ato violento, e o fato de a violência psicológica incidir fortemente nos alicerces da auto-estima feminina pode estar na base da maior lembrança deste tipo de abuso por parte das adolescentes atendidas nos serviços investigados ${ }^{34}$.

Dados semelhantes aos da nossa casuística são relatados por alguns autores ${ }^{35} \mathrm{em}$ relação à população de mulheres atendidas em serviços de emergência no Brasil, onde os profissionais referiram como agressor, predominantemente, um membro da família: parceiros íntimos, pais ou cuidadores, denotando certo paralelismo na tipologia da agressão - doméstica e familiar - entre os atendimentos prestados a mulheres, tanto em serviços de atenção básica, como o PSF, como nos emergenciais.

A abordagem metodológica visou privilegiar a obtenção de informações sobre a presença ou ausência de algum atendimento envolvendo episódio de violência, sendo o tipo de violência (psicológica, física ou sexual) durante a gestação adolescente, subjacentes ao desenho de estudo. Igualmente, não se constituiu objetivo do trabalho a busca de informações adicionais sobre o agressor, exceto a relação de parentesco ou afinidade com a adolescente atendida, resultando em uma limitação do escopo de abrangência do trabalho em relação a esta temática.

Entre os motivos alegados pelos profissionais para a ausência de encaminhamento e de notificação, o medo de retaliação por parte do agressor encontra paralelo em um trabalho já conduzido ${ }^{36}$, onde o lidar cotidiano com situações de VIF em comunidades consideradas violentas, se caracteriza pela discrição e prudência com respeito às "regras de convivência estabelecidas por um 'poder paralelo' que os domina através do terror $\mathrm{e}$ medo", forjando um comportamento peculiar das equipes do PSF, caracterizado pelo imobilismo, em nome da sobrevivência. Tal argumento, embora invocado com certa frequência para justificar a não-notificação dos casos de violência contra crianças e adolescentes por profissionais de saúde, não foi considerado fator relevante no trabalho empreendido por Gonçalves e cols. em estudos empreendidos no Ambulatório de Família do Instituto de Puericultura e Pediatria Martagão Gesteira /UFRJ ${ }^{32}$. 
Embora a notificação de casos de VIF seja preconizada pelo Estatuto da Criança e do Adolescente (ECA) como responsabilidade de todo e qualquer profissional da saúde que atenda adolescentes, os profissionais de nível superior demonstraram notificar e encaminhar casos de violência com maior frequência quando comparados a profissionais de nível médio, sendo a associação limítrofe do ponto de vista estatístico com o nível educacional da ocupação. Tal achado encontra apoio nas características predominantes do trabalho em equipe na atenção básica de saúde, avaliado para o caso brasileiro ${ }^{36}$, onde, embora o processo de trabalho no PSF sinalize para a possibilidade efetiva da autonomia do cuidado em saúde, o trabalho da equipe permanece centrado em relações hierárquicas, vinculadas aos profissionais com nível superior de escolaridade, com ênfase na lógica do trabalho médico. No caso particular de grupos prioritários, especialmente para o grupo materno-infantil, onde se insere a população de referência da nossa pesquisa, a enfermeira é quem realiza consultas, definidas pelas ações programáticas, voltadas para gestantes adolescentes.

Outros aspectos a considerar dizem respeito às possíveis explicações dos achados: os profissionais de nível superior teriam maior conhecimento sobre a temática, maior liberdade/autonomia para notificar e menor receio de retaliações por parte do agressor, até porque muitos profissionais de nível médio residem na própria comunidade.

Outro argumento utilizado pelos profissionais para a não notificação "Não ter provas da violência" se inscreve na vertente de dificuldades representadas pela cultura familiar ${ }^{32}$ que tende, na sociedade brasileira, a valorizar a salvaguarda da privacidade da vida familiar, e onde as intervenções são vistas como ofensivas à autoridade parental.

Embora não se tenha identificado artigos que comparem identificação e notificação de casos de VIF e nível educacional de membros da equipe profissional do PSF, estudo empreendido com pediatras em
Porto Alegre ${ }^{37}$ identificou que a maioria assinalou medo de envolvimento legal, enquanto conhecimento insuficiente sobre o tema mostrou associação estatisticamente significante com a não notificação.

Da análise da associação das variáveis de interesse, o estudo relacionou o aumento da idade e ter filhos como sendo associações estatisticamente significantes. Resultados semelhantes foram descritos por Pires et al. ${ }^{37}$, que encontraram maior frequência de relatos de notificação por parte de pediatras mais velhos e entre os que referiam ter filhos. Uma das possíveis explicações pode residir em atributos de ordem mais subjetiva como o fato de maior possibilidade evolutiva do processo de maturidade pessoal com o avançar da idade e ampliação da sensibilidade à questão, a partir da ativação de sentimentos de empatia e solidariedade, ao transportarem para o seu próprio imaginário o risco de acometimento de entes queridos.

Como destacado anteriormente, os resultados limítrofes em relação à significância estatística da análise de dados estão provavelmente relacionados com o pequeno número do total de profissionais que fazem parte da rede básica de assistência de saúde do município. Vale salientar por outro lado, a importância da investigação realizada, envolvendo a busca do conhecimento sobre a realidade da atuação profissional envolvendo situações de violência contra gestantes adolescentes, a partir do envolvimento da totalidade dos profissionais da rede de atenção básica. Os resultados encontrados são relevantes para a compreensão de eventos complexos, como a violência doméstica e familiar, em municípios de pequeno porte, como é o caso de S. Joaquim do Monte, no agreste pernambucano.

\section{Considerações finais}

Embora a abordagem de situações de violência intrafamiliar encontre obstáculos relacionados à obtenção de dados, devido aos inúmeros problemas associados à sua emergência e visibilidade ${ }^{38}$, a análise das 
ações (identificação, encaminhamentos e notificações) empreendidas por profissionais de saúde que trabalham na porta de entrada do sistema de saúde mostrou-se pertinente, representando um procedimento de aproximação deste fenômeno, complexo e multifacetado, sendo passível de fomentar o debate na área, ampliando as possibilidades de reflexão crítica sobre as diferentes formas de apreensão social do mesmo.

Os dados levantados remetem à discussão que vem sendo travada, concernente à necessidade de compreensão dos mecanismos que modulam a conduta profissional, onde o perfil sociodemográfico dos profissionais envolvidos, o medo e a falta de informações sobre responsabilidades na atenção de saúde a gestantes adolescentes tornam-se elementos emblemáticos na superação dos obstáculos para o aprimoramento do sistema de registro das notificações, no contexto do PSF.

Outro aspecto que aqui se defende se refere à necessidade de dar destaque à necessidade de instrumentalização do olhar por parte dos profissionais dos serviços de atenção à saúde, uma vez que a percepção da violência passa pelo reconhecimento do problema: capacitação técnica das equipes com melhoria da orientação prestada aos profissionais, desde as noções legais (definição de caso suspeito e confirmado de violência, situações que devem ser notificadas, fluxos de atendimento, etc.); elaboração e discussão de manuais de orientação acerca da questão; reforço da atuação em rede, especialmente com os Conselhos Tutelares, integrando-as aos demais serviços envolvidos no atendimento às vítimas de violência.

\section{Colaboradores}

MT Oliveira participou da concepção, trabalho de campo, análise dos dados e redação do artigo. ABM Ishigami e RMM Nascimento contribuíram com a avaliação de consistência do banco, análise dos dados e a escrita do artigo. I Samico contribuiu com revisão final do artigo.

\section{Agradecimentos}

Ao Instituto de Medicina Integral Prof. Fernando Figueira - IMIP, Universidade de Pernambuco/UPE, Núcleos de Prevenção da Violência e Promoção da Saúde da UPE e NUSP/UFPE, Espaço Família - Serviços Jurídicos e Terapêuticos, Secretaria de Saúde de S. Joaquim do Monte/ PE e à FACEPE/ MS pelo apoio dado para que a pesquisa pudesse ocorrer.

\section{Referências}

1. Minayo MCS, Souza ER 1998. Violência e saúde como um campo interdisciplinar e de ação coletiva. História, Ciências, Saúde 1998; IV: 513-31.

2. Moura ATMS, Reichenheim ME. Estamos realmente detectando violência familiar contra a criança em serviços de saúde? A experiência de um serviço público do Rio de Janeiro, Brasil. Cad Saúde Pública 2005; 21(4): 1124-33.

3. Paim JS, Costa HOG, Vilasbôas ALQ. Política pública e controle da violência: um estudo de caso na cidade de Salvador, Bahia, Brasil. Cad Saude Pública 2009; 25(3): 485-94.

4. Minayo MCS. Violência e saúde. Rio de Janeiro: Fiocruz; 2006
5. Day VP, Telles LEB, Zoratto PH, Azambuja MRF, Machado DA, Silveira MB, et al. Violência doméstica e suas diferentes manifestações. Rev Psiquiatr Rio Gd Sul 2003; 25(1): 9-21.

6. Mascarenhas MDM, Malta DC, Silva MMA, Lima CM, Carvalho MGO, Oliveira VLA. Violência contra a criança: revelando o perfil dos atendimentos em serviços de emergência, Brasil, 2006 e 2007. Cad Saúde Pública 2010; 26(2): 347-57.

7. Saliba O, Garbin CAS, Garbin AJI, Dossi AP. Responsabilidade do profissional de saúde sobre a notificação de casos de violência doméstica. Rev Saúde Pública 2007; 41(3): 472-7. 
8. Minayo MCS. Violência contra crianças e adolescentes: questão social, questão de saúde. Rev Bras Saúde Matern Infant 2001; 1:91-102.2001 In: Bazon MR. Violências contra crianças e adolescentes: análise de quatro anos de notificações feitas ao Conselho Tutelar na cidade de Ribeirão Preto, São Paulo, Brasil. Cad Saúde Pública 2008; 24(2): 323-32.

9. Garcia-Moreno C, Jansen HAFM, Ellsberg M, Heise L, Watts C. Prevalence of intimate partner violence: findings from the WHO multi-country study on women's health and domestic violence. Lancet 2006; 368: 1260-9.

10. Nasir K, Hyder AA. Violence against pregnant woman in developing countries: review of evidence. Eur J Public Health 2003; 13(2): 105-7.

11. D'Oliveira AFPL, Schraiber LB, França-Junior I, Ludermir AB, Portella AP, Diniz CS et AL. Fatores associados à violência por parceiro íntimo em mulheres brasileiras. Rev Saúde Pública 2009; 43(2): 299-310.

12. Martins CBG, Jorge MHP. A violência contra crianças e adolescentes: características epidemiológicas dos casos notificados aos Conselhos Tutelares e programas de atendimento em município do Sul do Brasil, 2002 e 2006. Epidemiol Serv Saúde 2009; 18(4): 315-34.

13. Kaye DK, Mirembe FM, Bantebya G, Johansson A, Ekstrom AM. Domestic violence during pregnancy and risk of low birthweight and maternal complications: a prospective cohort study at Mulago Hospital, Uganda. Trop Med Int Health 2006; 11(10): 1576-84.

14. D'Oliveira AFPL, Schraiber LB. Violência de gênero como questão de saúde pública: a importância da formação de profissionais. J Rede Saude 1999; 19: 3-4.

15. Ishida VK. Estatuto da Criança e do Adolescente. Doutrina e Jurisprudência. 9a ed. São Paulo: Atlas; 2008.

16. Gonçalves HS, Ferreira AL. Dificuldades da notificação da violência doméstica contra crianças e adolescentes. In: Mello AC (coord.). Anais do III Congresso Iberoamericano de Psicologia Jurídica. São Paulo: Universidade Presbiteriana Mackenzie; 2000.

17. Souza ER, Ribeiro AP, Penna LHG, Ferreira AL, Santos NC, Tavares CMM. O tema violência intrafamiliar na concepção dos formadores dos profissionais de saúde. Ciênc Saúde Coletiv 2009; 14(5): 1709-19.

18. Reichenheim ME, Dias AS, Moraes CL. Co-ocorrência de violência física conjugal e contra filhos em serviços de saúde. Rev Saúde Pública 2006; 40(4): 595-603.

19. Luna GLM, Ferreira RC, Vieira LJES. Notificação de maus-tratos em crianças e adolescentes por profissionais da Equipe Saúde da Família. Ciênc saúde coletiva 2010; 15(2): 481-91.

20. Presidência da República - Casa Civil. Lei no 11.340 , de 7 de agosto de 2006 - Lei Maria da Penha. Disponível em http://www.planalto.gov.br/ccivil_03/_ato200406/2006/ lei/111340.htm [Acessado em maio de 2010]
21. Schraiber LB, D’Oliveira AFPL, Hanada H, Figueiredo W, Couto M, Kiss L, Durand J, Pinho A. Violência vivida: a dor que não tem nome. Interface (Botucatu) 2003; 7(12): 41-54.

22. Murofuse NT, Rizzotto MLF, Muzzolon ABF, Nicola AL. Diagnóstico da situação dos trabalhadores em saúde e o processo de formação no polo regional de educação permanente em saúde. Rev Latino-Am Enfermagem 2009; 17(3): 314-20.

23. Jaramillo DE, Uribe TM. Rol del personal de salud en la atención a las mujeres maltratadas. Invest Educ Enferm 2001; XIX(1): 38-45.

24. Kodato LAB , Nakama L. Pesquisa em saúde: metodologia quantitativa ou qualitativa? Health's research: quantitative or qualitative methodology? Rev Espaço para a Saúde (Londrina) 2006; 8(1): 34-5.

25. Plano Municipal de Saúde 2006-2009, São Joaquim do Monte/ PE; 2006. 59p. [mimeo]

26. Schraiber LB, D’Oliveira AFPL, Júnior IF, Diniz S, Portella $\mathrm{AP}$, Ludemir $\mathrm{AB}$ et al. Prevalência da violência contra a mulher por parceiro íntimo em regiões do Brasil. Rev Saúde Pública 2007; 41(5): 797-807.

27. Lilienfeld AM, Lilienfeld DE. Foundations of Epidemiology. $2^{\text {a }}$ ed. Oxford University Press: New York; 1980.

28. Barreto AP. Terapia Comunitária passo a passo. $3^{\text {a }}$ ed. [revisada] Fortaleza: Gráfica LCR; 2008.

29. Lopes MJM, Leal SMC. A feminização persistente na qualificação profissional da enfermagem brasileira. Cadernos Pagu 2005; (24): 105-25.

30. Gil CRR. Formação de recursos humanos em saúde da família: paradoxos e perspectivas. Cad Saúde Pública 2005; $21(2): 490-8$.

31. Gonçalves HS, Ferreira AL. A notificação da violência intrafamiliar contra crianças e adolescentes por profissionais de saúde. Cad Saúde Pública 2002; 18(1): 315-9.

32. Moura ATMS, Moraes CL, Reichenheim ME. Detecção de maus-tratos contra a criança: oportunidades perdidas em serviços de emergência na cidade do Rio de Janeiro, Brasil. Cad Saúde Pública 2008; 24(12): 2926-36

33. Durand JG; Schraiber LB. Violência na gestação entre usuárias de serviços públicos de saúde da Grande São Paulo: prevalência e fatores associados. Rev Bras Epidemiol 2007; 10(3): 310-22

34. Zelaski M et cols. Violência entre parceiros íntimos e consumo de álcool. Rev Saúde Pública 2010; 44(1): 53-9.

35. Mascarenhas MDM, Silva MMA, Malta DC, Moura L, Macário EM, Gawryszewski VP et al. Perfil epidemiológico dos atendimentos de emergência por violência no Sistema de Serviços Sentinelas de Vigilância de Violências e Acidentes (Viva) - Brasil; 2006. Epidemiol Serv Saúde (Brasília) 2009; 18(1): 17-28. 
36. Santos NC. Práticas Sanitárias Frente à Violência Intrafamiliar no Âmbito do PSF - Um Estudo de Caso [tese de doutorado]. Rio de Janeiro: ENSP/FIOCRUZ; 2005.

37. Pires JM, Goldani MZ, Vieira EM, Nava TR, Feldens L, Castilhos K, et al. Barreiras, para a notificação pelo pediatra, de maus-tratos infantis. Rev Bras Saúde Matern Infant 2005; 5 (1): 103-8.
38. Minayo MCS. A violência social sob a perspectiva da saúde pública. Cad Saúde Pública 1994; 10(S1): S7-18.

Recebido em: 24/06/10

Versão final apresentada em: 02/11/10 Aprovado em: 06/04/11 